\title{
Penser le nouveau paradigme de l'hydrologie urbaine : biomimétisme, éco-phénoménologie, et gestion intégrée
}

\author{
Henry DICKS
}

IrPhil, Université Jean Moulin Lyon 3, 18 rue Chevreul, 69007 Lyon-e-mail : henryjdicks@gmail.com

\begin{abstract}
RÉSUMÉ. - Cet article propose une analyse historique et épistémologique de la problématique d'un « nouveau paradigme » pour l'hydrologie urbaine. A partir d'une étude de diverses visions de ce nouveau paradigme [Todd et Todd, 1993 ; Narcy, 2004 ; Novotny et Brown, 2007 ; Andrieu et al., 2010 ; Chocat, 2013], nous dégageons trois éléments qui semblent faire plus ou moins consensus : le biomimétisme, l'éco-phénoménologie, et la gestion intégrée. Le biomimétisme propose de mimer les flux hydrologiques d'écosystèmes naturels. L'éco-phénoménologie se focalise sur la question de la manifestation phénoménale de l'eau : réouverture de cours d'eau urbains, rétention des eaux de surface, purification locale de l'eau, mesures pédagogiques, etc. Et la gestion intégrée propose de gérer de manière concertée les divers moments du cycle hydrologique urbain en favorisant la coopération entre les divers acteurs et parties prenantes. Le fil conducteur qui nous permettra de rassembler ces trois éléments en une unité épistémique cohérente sera l'argument suivant : le paradigme actuel reste imprégné de l'imaginaire organiciste de la ville qui a accompagné la conception des premiers grands réseaux hydrauliques urbains au XIX ${ }^{\mathrm{e}}$ siècle; or, en raison des problèmes écologiques qu'entraîne cet imaginaire, le paradigme actuel est entré en crise et un nouvel imaginaire et de fait un nouveau paradigme - est en train d'émerger : " la ville comme une forêt » [Braungart et McDonough, 2009, p.139].
\end{abstract}

Mots-clés : villes biomimétiques, philosophie environnementale, GIRE, urbanisme, histoire urbaine.

\section{A new paradigm for urban hydrology: biomimicry, eco-phenomenology, and integrated management}

\begin{abstract}
This article puts forward a historical and epistemological analysis of the idea that urban hydrology requires a "new paradigm". Through a study of various visions of this new paradigm [Todd and Todd, 1993; Narcy, 2004; Novotny and Brown, 2007; Andrieu et al., 2010; Chocat, 2013], I outline three elements that seem relatively consensual: biomimicry, eco-phenomenology, and integrated management. Biomimicry enables us to think about how we can imitate or take inspiration from the hydrological flows of natural ecosystems. Eco-phenomenology - a philosophical approach rarely advocated explicitly by urban hydrologists - draws attention to the phenomenological manifestation of water in the city: daylighting urban streams and rivers, surface storage, local purification, pedagogical measures, etc. And integrated management makes it possible to manage the various moments of the urban hydrological cycle in a concerted manner, integrating bottom-up initiatives and lateral cooperation between the various actors and stakeholders of the urban water cycle. The guiding thread that holds these three elements together in a coherent epistemic framework is the following argument: the current paradigm remains beholden to the organicist imaginary of the city that accompanied the conception of the first hydraulic networks of the nineteenth century [Chocat, 1997; Narcy, 2004; Illich, 2005; Bertrand-Krajewski, 2006; Picon, 2014]; however, as a result of the ecological problems that this imaginary entails, the current paradigm has entered a period of crisis and a new imaginary - and thus also a new paradigm - is in the process of taking its place: the biomimetic idea of imagining the city like an ecosystem [Newman and Jennings, 2008 ; Schuiten, 2009 ; Despommier, 2011] and more specifically "like a forest" [Braungart and McDonough, 2009, p.139]. From this perspective, the imaginary of the "urban organism" [Harrouel 1977] may be described as what the French philosopher, Paul Ricoeur [1975], calls a "dead metaphor": a metaphor that continues to structure our thoughts and deeds but which we no longer recognize as a metaphor. What is instead required, then, is what Ricoeur calls a "living metaphor" - the metaphor of the city as a forest - capable of stimulating the imagination and integrating activity in such a way that a "new paradigm" for the city - and thus also for urban hydrology - may finally emerge.
\end{abstract}

Key-words: biomimetic cities, environmental philosophy, IWRM, urbanism, urban history. 


\section{INTRODUCTION}

Face au constat de la pollution des eaux et des sols urbains, de la raréfaction de la ressource en eau, d'inondations en milieu urbain dues parfois à l'imperméabilisation des sols, et de la nécessité de mitiger et d'adapter au changement climatique ${ }^{1}$, les appels à un " nouveau paradigme " de l'eau urbaine, et notamment des eaux pluviales, se font de plus en plus entendre [Narcy, 2004 ; Novotny et Brown, 2007 ; Andrieu et al., 2010 ; Chocat, 2013]. En effet, un consensus semble être en train d'émerger selon lequel il ne suffit pas de modifier et d'améliorer le système actuel - souvent qualifié de « tout-à-l'égout »-, mais d'aller plus loin et proposer un «nouveau paradigme » à la hauteur de la transition écologique à venir. Certes, les avantages $\mathrm{du}$ système actuel, notamment la disponibilité générale d'eau potable et les bénéfices apportés au niveau de la santé publique, sont à conserver [Brown, 2007], mais cela ne veut dire qu'ils sont forcément à assurer par les mêmes dispositifs qu'aujourd'hui.

En s'inspirant des diverses tentatives de penser ce nouveau paradigme de l'eau urbaine, cet article propose une analyse historique et épistémologique dont l'objectif est de synthétiser trois de ses éléments clés : le biomimétisme, l'écophénoménologie, et la gestion intégrée. Parmi ces trois éléments, le biomimétisme nous paraît particulièrement important. En effet, la thèse principale de cet article est que le passage à un nouveau paradigme de l'eau urbaine nécessitera un changement important de notre « imaginaire urbain » : alors que l'imaginaire urbain qui dominait lors de la construction des réseaux hydrauliques urbains au $\mathrm{XIX}^{\mathrm{e}}$ siècle était celui de l'« organisme urbain » et plus précisément celui de la ville pensée comme un « corps animal », nous pensons que l'imaginaire urbain dont a besoin le nouveau paradigme est celui de l'« écosystème urbain » et plus précisément celui de la ville pensée comme "une forêt ». L'importance du biomimétisme tient donc au fait qu'il peut nous fournir une nouvelle image et un nouvel imaginaire de l'urbain capable de rassembler en une unité cohérente les divers éléments du nouveau paradigme de l'hydrologie urbaine.

\section{BIOMIMÉTISME}

Le biomimétisme est une "science nouvelle » qui propose des innovations techniques qui imitent ou s'inspirent de modèles naturels. Ainsi, au lieu de créer des produits et des systèmes artificiels qui fonctionnent d'une manière différente de - et généralement opposée à - la nature, il s'agit de suivre l'exemple durable et fertile de celle-ci. Dans le livre fondateur de J. Benyus, Biomimicry : Innovation Inspired by Nature [1997], cette approche se décline en plusieurs champs : l'agriculture, l'énergie, la manufacture, la médecine, l'informatique, et le commerce. Plus récemment, un nouveau champ - plus ou moins absent du livre de J. Benyus - a émergé : la ville biomimétique [Newman and Jennings, 2008 ; Braungart and McDonough, 2009 ; Schuiten, 2009 ; Despommier, 2011 ; Beatley, 2011 ; Perdersen Zari, 2015]. Dickson Despommier, dont la réflexion sur les « fermes verticales » se situe dans une tentative de penser la ville

1. Le changement climatique a tendance à exacerber les phénomènes météorologiques extrêmes, notamment les périodes de pluie forte, qui augmentent le risque d'inondations, et les sécheresses, qui peuvent provoquer des pénuries d'eau et sont également associées à des températures extrêmes. Pour les villes côtières, la montée du niveau de la mer peut également poser des problèmes d'inondations et de salinisation des sols. biomimétique, explique la logique qui sous-tend ce concept comme suit : «En comprenant les éléments fondamentaux qui permettent à des ensembles de plantes et d'animaux de s'organiser en des réseaux interdépendants appelés écosystèmes, nous pouvons mieux voir comment une ville pourrait être reconçu pour mimer ce processus » (Despommier, 2011, p.16, nous traduisons). Conformément à cette logique, $\mathrm{V}$. Novotny et P. Brown proposent comme élément clé du nouveau paradigme de l'hydrologie urbaine l'imitation des flux hydrologiques de "systèmes naturels pré-anthropisés» (predevelopment natural systems). Ainsi, au lieu de canaliser les eaux pluviales dans des tuyaux afin de s'en débarrasser le plus vite possible (fast conveyance), on ferait mieux, selon eux, de mimer le fonctionnement hydrologique d'un écosystème naturel, notamment en rouvrant des cours d'eaux urbains, en multipliant des surfaces perméables, et en ralentissement l'écoulement des eaux de surface [Novotny and Brown, 2007, xix].

Une lecture rapide de l'histoire de la pensée permet de relativiser la nouveauté prétendue du biomimétisme urbain. Il suffit de regarder l'industrialisation et l'expansion des villes européennes aux $\mathrm{XVIII}^{\mathrm{e}}$ et $\mathrm{XIX}^{\mathrm{e}}$ siècles pour constater qu'une image biologique a joué un rôle important dans la conception des grands réseaux urbains : celle de l'« organisme urbain » [Harrouel, 1977 ; Picon, 2014]. Sous l'emprise de cette image - associée en France surtout à la "physiologie sociale » de Saint-Simon et des Saint-Simoniens -, les conduites d'eau potable étaient conçues par analogie avec les « artères » et les conduites d'eaux usées et pluviales avec les « veines» [Chocat, 1997 ; Narcy, 2004; Illich, 2005 ; Bertrand-Krajewski, 2006 ; Thual, 2011 ; Picon, 2014]. Ceci est conforme aux analyses de Judith Schlanger [1995] et de Pierre Musso [1997], qui montrent que l'imaginaire organiciste connaissait son âge d'or dès la fin du XVIII ${ }^{\mathrm{e}}$ siècle et puis tout au long du $\mathrm{XIX}^{\mathrm{e}}$ siècle, période qui correspondait en Europe à la conception des grands réseaux urbains, y compris les réseaux hydrauliques.

D’une perspective écologique, il existe (au moins) trois problèmes majeurs qui découlent de l'imaginaire de l'« organisme urbain » et, plus précisément, de la ville pensée " comme un corps animal ». Un premier problème concerne le statut écosystémique de l'animal. D'un point de vue écologique, un animal n'est pas un écosystème, mais un consommateur dans lequel aucune production au sens écologique (auto-tropisme) n'a lieu. En tant que tel, il dépend de la production (plantes...) et de la décomposition (champignons...) qui ont lieu dans son environnement. Ainsi, lorsque la ville est pensée comme un corps animal, sa taille importante mène à une consommation massive de produits puisés dans son environnement proche ou lointain, y compris l'eau, ainsi que le déversement d'énormes quantités de déchets au-delà de ses bords.

Un deuxième problème concerne la vision mécaniste de l'organisme qui dominait la pensée scientifique et technique de l'époque. Selon l'analyse de J. Schlanger [1995, p.28], la physiologie moderne, qui remonte notamment au médecin anglais, Harvey, tirait ses images de la technique mécanique, particulièrement les techniques hydrauliques. Ainsi, même si les urbanistes du XIX $\mathrm{X}^{\mathrm{e}}$ siècle - Chadwick à Londres [Harpet, 1998], Haussman à Paris [Picon, 2014], etc. - concevaient la ville comme un organisme, cet organisme était lui-même conçu comme une machine composée de tuyaux, de pompes, de valves, de pistons, et d'autres objets mécaniques. Cette combinaison d'une vision mécaniste de l'organisme et d'une vision organiciste de la ville favorisait des villes où proliféraient des objets et des réseaux mécaniques fabriqués à 
base de minéraux (béton, métal, verre, plastique...), laissant peu de place à toute forme de vie non-humaine, l'exception principale étant les boulevards arborés et les parcs, souvent octroyés le rôle de « poumons de la ville » [Mumford, 1961, p.475].

Le rapport entre l'organisme urbain et la montée de l'hygiénisme présente un troisième problème. L'imaginaire de l'organisme urbain a pour conséquence non seulement que l'eau se voit canalisée dans des tuyaux de sorte qu'elle circule en permanence et ne peut donc "stagner », mais la plupart des êtres vivants qui arrivent à pénétrer dans l' " organisme urbain », notamment les rats, les pigeons, et les mauvaises herbes, assument le rôle de pestes, de parasites, ou de vecteurs de maladies à traiter par divers dispositifs d' " immunisation» (pesticides, vaccins, expulsions, etc.). Un tel constat ne veut pas dire que le mouvement hygiéniste n'a pas apporté des bénéfices à la santé public, mais il soulève toutefois la possibilité suivante : la conception organiciste de la ville va de pair avec la manifestation $\mathrm{du}$ vivant non-humain en milieu urbain avant tout comme élément étranger indésirable, que ce soit comme peste, parasite, ou vecteur de maladies.

S'il est vrai que nous, aujourd'hui, sommes les héritiers de cet imaginaire de l'organisme urbain, une décision importante semble se présenter : i) abandonner cet imaginaire pour penser la ville en tant que telle, et non pas de façon métaphorique, c'est-à-dire en tant qu'autre chose ; ou ii) développer un nouvel imaginaire de l'urbain qui nous permettrait de réaliser l'objectif principal de l'urbanisme du $\mathrm{XXI}^{\mathrm{e}}$ siècle : la transition écologique vers des villes durables. En ce qui concerne la première possibilité, le simple fait que les villes que nous a léguées le $\mathrm{XIX}^{\mathrm{e}}$ siècle étaient conçues comme des organismes indique une première difficulté épistémique : qu'est-ce qu'une ville " en tant que telle » ? Certes, on pourrait tenter de la définir comme un milieu anthropisé qui contient une population et une densité minimales d'habitants humains, et, à partir de là, étudier les problèmes - hydrologiques ou autres - que connaissent les villes contemporaines dans le but d'essayer de les remédier de façon ponctuelle. Or, une telle approche risque de ne pas être à la hauteur d'un véritable changement de paradigme. En effet, sans une vision plus concrète d'où viennent, et de ce que sont, nos villes actuelles, il sera difficile de passer à des villes véritablement différentes et on risquera de rester dans le paradigme de l'organisme urbain, lequel, dès lors, aurait le statut de ce que P. Ricoeur [1975] appelle une «métaphore morte $",{ }^{2}$ capable en tant que telle de déterminer même nos actions les plus pragmatiques par sa «fécondité occulte» [ibid., p.371].

La deuxième possibilité correspond à ce que proposent les partisans de la ville biomimétique : la ville devrait désormais être imaginée et conçue comme un écosystème. Or, en dehors de toute pensée explicitement «biomimétique » ou « bio-inspirée », très nombreux sont ceux aujourd'hui qui n'hésiteraient pas à décrire la ville comme un écosystème. Une telle description semble même incontestable dans la mesure où toute ville comprend une multiplicité d'êtres vivants en interaction à la fois entre eux et avec leur milieu abiotique. D'autre part, même si nos villes contemporaines correspondent à cette vision minimaliste de ce que c'est qu'un écosystème, d'autres système naturels, tels les

2. Selon P. Ricoeur, une « métaphore morte » est une métaphore qui autrefois était « vive », et, en tant que tel, animait et excitait l'imaginaire des gens, mais qui peu à peu s'est intégrée dans la pensée et dans les faits à un tel point qu'on n'en est plus conscient en tant que métaphore. intestins des mammifères ${ }^{3}$ ou les nids d'insectes sociaux (ruches, termitières...), y correspondent aussi. Si, par contre, on réserve le concept d'écosystème à des systèmes alimentés par l'énergie renouvelable (solaire principalement), où des nutriments circulent autour d'un réseau complexe et relativement fermé, et qui sont composés d'une grande diversité d'espèces (végétales, animales, fongiques, etc.), les villes modernes ne sauraient guère être désigné par le mot « écosystème ». Cela ne veut pas dire qu'il y a contradiction entre la description de la ville comme un écosystème et le souhait que la ville soit - ou soit comme - un écosystème. En effet, comme dans le cas de l'imaginaire de l'« organisme urbain », l'imaginaire de l' « écosystème urbain » suggère un développement plus ou moins conforme à cette image, c'est-à-dire un développement qui rendrait la ville plus proche de notre compréhension actuelle de ce que c'est qu'un écosystème naturel.

Dans notre analyse de l'organisme urbain, nous avons vu que la ville n'était pas conçue comme un organisme au sens abstrait, mais plutôt comme un corps animal, d'où l'imagerie d'artères, de veines, de poumons, du système nerveux, etc. Tenant compte de ceci, il semble important de se demander si un type particulier d'écosystème peut fournir une nouvelle "métaphore vive » de l'urbain ? Dans leur livre influent, Cradle to cradle, les biomiméticiens, M. Braungart et W. McDonough, proposent l'exercice suivant : «Imaginez un bâtiment comme un arbre et une ville comme une forêt » [2009, p.139, nous traduisons]. Dans ce qui suit, nous allons poursuivre cet exercice de l'imagination à partir d'une brève analyse écosystémique d'une forêt.

D'un point de vue écosystémique, un aspect très important d'une forêt - auquel J. Benyus [1997, p.248-254] est particulièrement attentive - est son statut d'écosystème de Type III, dont la végétation caractéristique est l'arbre, et non pas la plante annuelle (écosystème de Type I) ou la plante vivace (écosystème de Type II). Or, à propos de ces « écosystèmes matures », J. Benyus observe qu'ils « font tout ce que nous voulons faire » [ibid., p.248, nous traduisons] : le recyclage de nutriments en boucles plus ou moins fermées, l'usage efficient d'énergie solaire, le maintien d'une biodiversité complexe, participation à la régulation climatique, et ainsi de suite. ${ }^{4}$ Ainsi, alors que les villes contemporaines utilisent l'eau de manière linéaire et souvent polluante, la traitent et la transportent souvent à l'aide d'énergies fossiles ou nucléaires, ne s'intéressent à son interaction qu'avec une seule espèce (ou presque), et méconnaissent son rôle dans la régulation climatique, une ville biomimétique, modelée d'après ou inspirée d'une forêt, utiliserait l'eau de manière plus circulaire, la traiterait et la transporterait à l'aide de la gravité et de l'énergie solaire, la laisserait interagir avec une grande pluralité d'espèces, et en tirerait bénéfice pour mieux réguler le climat (évapotranspiration, etc.).

Il existe néanmoins un aspect important de toute ville qui complique cette vision biomimétique : les êtres humains. Que deviennent les habitants humains d'une ville

3. Il existe dans les intestins des mammifères une multiplicité de bactéries qui vivent à la fois en interaction entre elles, aves les cellules du corps humain, et avec leur milieu abiotique (eau, oxygène, etc.).

4. Un rapport récent, co-écrit par le Biomimicry Group de J. Benyus et la société d'architectes, HOK (2013), part du constat que de nombreuses régions très peuplées du monde, comme l'est de la Chine, l'est des Etats-Unis, et le nord de l'Europe, partagent à peu près le même biome - la forêt tempérée - pour ensuite proposer diverses manières dont on peut mimer ou s'inspirer de ce biome pour résoudre des problèmes humains. Néanmoins, si ce rapport donne de nombreux exemples qui concernent la ville (transports urbains, etc.), elle ne théorise pas le concept de « ville biomimétique » en tant que tel. 
biomimétique ? Ceci est particulièrement difficile à dire dans la mesure où le concept même de ville biomimétique préconise, pour reprendre l'expression de V. Novotny et P. Brown, l'imitation d'un « système naturel pré-anthropisé » [2007, xix, nous traduisons], c'est-à-dire un système d'où les êtres humains sont absents. ${ }^{5} \mathrm{~A}$ la lumière de ce constat, il nous semble important de noter qu'avant le processus qu'on appelle " hominisation », la surface de la terre était majoritairement couverte de forêts, et que les ancêtres d'homo sapiens habitaient ces forêts, et plus précisément dans les arbres. Or, les êtres humains que nous sommes ne sont pas seulement "descendus" de nos ancêtres primates; ils sont aussi « descendus » des arbres pour s'installer dans, et aussi pour défricher, des "clairières ». En effet, c'était dans des clairières qu'ils pouvaient pratiquer la chasse, cueillir et cultiver des plantes comestibles, bâtir des logements, maîtriser l'usage du feu, regarder le ciel et les étoiles, enterrer les morts, et raconter des histoires qui donnaient un sens à leur existence. L'émergence de l'humanité, ainsi que son développement à travers le temps, semble donc intimement liée à l'existence et à l'expansion de ses "clairières ", comme l'a déjà proposé Vico dans La science nouvelle [1725/1993], et d'une certaine façon aussi Heidegger [1976], qui prend la "clairière » comme image de l'ouverture spécifiquement humaine à l'être des choses, c'est-à-dire notre capacité unique de comprendre les choses « comme ceci» ou « comme cela ».

Cette réflexion sur les clairières semblera sans doute assez éloignée des enjeux plus opérationnels auxquels s'intéressent les hydrologues urbains. Il n'empêche que l'image de la " clairière " nous paraît un élément essentiel pour éviter certains dangers présents dans la tentative de concevoir une ville « comme une forêt ». Une ville composée de bâtiments comme des arbres et d'autres aspects fonctionnels d'un écosystème forestier n'est pas encore une ville au sens d'une polis ; il lui manque des " clairières ", c'est-à-dire des espaces ouverts et publics («agora» en grec), où les choses peuvent $s$ 'ouvrir à l'échange, à l'interprétation, et au débat, mais aussi se retirer par diverses formes de catégorisation (du grec « cata- », signifiant « contre »+ « agora », signifiant « espace ouvert» et par extension « lieu d'assemblée public »), telles l'attribution d'un droit de propriété, la fixation conceptuelle, ou la décision exécutive. L'image de la « clairière " nous paraît donc essentielle pour éviter que le principe biomimétique de prendre la nature « comme mesure " [Benyus, 1997] - et donc comme point de repère qui permet de juger la « justesse » (rightness) des innovations techniques et organisationnelles - transforme la ville en un système totalitaire et inhumain. En effet, afin que les êtres humains puissent être véritablement humains, ils ne sauraient être réduits à des éléments objectifs dans un écosystème urbain dont le principe indiscutable - car commandé par " la nature »- serait l'imitation d'un écosystème naturel de type III. De ce point de vue, la question de quelle innovation est juste ne saurait être déterminée par les propriétés objectives d'un écosystème : au contraire, il faudrait interpréter et débattre de ce qui est « juste » dans cet espace ouvert et public qui distingue l'humain de l'animal et que la « ville forêt » nous invite à penser en tant que « clairière ».

\footnotetext{
5. On pourrait traduire et interpréter autrement la notion de «predevelopment natural ecosystem ». Par exemple, si on estimait que la notion de « développement » caractérise seulement le monde moderne issu de l'occident, on pourrait peut-être dire qu'il existe des "predevelopment natural ecosystems » composé en partie d'êtres humains, lesquels peuvent ne pas posséder le concept d'une nature séparée du monde humain (voir Descola 2006).
}

\section{III. ÉCO-PHÉNOMÉNOLOGIE}

Le deuxième élément clé du nouveau paradigme de l'hydrologie urbaine peut être abordé par ce que nous appelons l' "éco-phénoménologie ». L'éco-phénoménologie est un courant philosophique qui étudie le rapport vécu et expérientiel qu'entretiennent les êtres humains avec leur environnement naturel [Brown and Toadvine, 2003]. Rarement réclamée de façon explicite par les hydrologues urbains, l'éco-phénoménologie nous semble toutefois nécessaire pour bien comprendre un deuxième élément important du nouveau paradigme : le principe de rendre l'eau visible (où « visibilité 》 est à comprendre au sens large de toute manifestation phénoménale). Ainsi, les ingénieurs écologiques, J. Todd et N. J. Todd, expliquent comment l'eau peut « refaire surface » pour redevenir une «partie visible» de l'infrastructure urbaine, ayant plusieurs rôles à jouer au sein de la communauté [1993, p.98-100]. De façon similaire, P. Brown critique les «infrastructures invisibles " gérée de manière centralisée pour mettre l'accent sur la nécessité d'engager des « citoyens partenaires » [2007]. Et H. Andrieu et al. parlent de "rendre l'eau transparente », expression dont le sens se précise dans le passage suivant:

Au cours des dernières décennies, l'eau a disparu de la ville pour être cantonnée aux réseaux souterrains d'évacuation auxquels sont reliées les surfaces imperméabilisées. L'eau retrouve actuellement une nouvelle place, comme l'illustrent les projets d'aménagement des rivières urbaines ou de réouverture de certains cours d'eau urbains. Les nouvelles pratiques qui visent à améliorer la transparence de la ville pour le cycle de l'eau, telles que les dispositifs de retenue et d'infiltration des eaux pluviales à la source contribuent également à réintroduire l'eau dans la ville à la vue et à l'esprit des habitants. [Andrieu et al., 2010, p.138]

A partir d'une critique généralisée du " tout-à-l'égout » et des « infrastructures invisibles » émerge donc l'appel à « sortir l'eau des tuyaux » [Narcy 2004], les bénéfices recherchés étant non seulement écologiques, mais aussi économiques, sociaux, culturels et esthétiques. Par ailleurs, il nous semble également possible d'intégrer dans cette perspective éco-phénoménologique le concept d'origine australienne de « conception urbaine sensible à l'eau » (Water Sensitve Urban Design - WSUD), cette sensibilité étant un indice important d'un nouveau rapport phénoménologique entre les citadins et l'eau, celle-ci ayant dès lors le statut d'élément central du paysage urbain. ${ }^{6}$

Le principe de rendre l'eau visible va de pair avec la métamorphose de l'« organisme urbain » en une «ville forêt ». En effet, lorsque la ville était pensée par analogie avec l'organisme, il était tout à fait normal que la circulation des eaux soit cachée dans des tuyaux sous des surfaces imperméables. L'image de la «ville forêt », en revanche, va de pair avec la « redécouverte » des eaux urbaines: certaines « veines" se transforment en ruisseaux et rivières, les sols redeviennent perméables, et, tout comme les arbres, les bâtiments purifient et recyclent l'eau sur place [Braungart et McDonough 2009, p.90], parfois à l'aide de nouvelles techniques d'épuration biomimétiques, comme les «machines vivantes » de N.J. Todd et J. Todd [1993]. De plus, ce n'est pas seulement l'eau qui serait ainsi « redécouverte": alors que l'« organisme urbain » a tenté d'éliminer de la ville la plupart des formes de vie non-humaine la « ville forêt » rassemblerait

6. Pour une étude plus détaillée de l'approche éco-phénoménologique, voir notre article «A Phenomenological Approach to Water in the City : Towards a Policy of Letting Water Appear» [Dicks, 2014]. 
des phénomènes biologiques divers, grâce à ses cours d'eaux redécouverts, ses berges aménagées, ses toitures végétalisées, ses fermes verticales, et autres.

Par ailleurs, dans une telle "ville forêt », le citoyen ne serait plus réduit à un simple acheteur d'eau potable, aux yeux de qui l'eau n'existe de façon positive qu'en tant que produit de consommation, toute autre manifestation de l'eau étant intrinsèquement négative (précipitations gênantes, inondations dangereuses, fuites dommageables...). En effet, alors que dans l'« organisme urbain », le citoyen est réduit à un simple composant d'un système plus large de consommation - une cellule consommatrice dans un méga-organisme consommateur -, dans la « ville forêt » l'eau pourrait revêtir une multiplicité de formes phénoménales pour le citoyen : l'aménagement des berges d'un fleuve créerait un lieu de promenade, de sociabilité, et de rêverie poétique ; l'eau de pluie serait récupérée et stockée sur place pour des usages divers, notamment l'arrosage d'espaces verts ou l'agriculture urbaine ; les eaux grises seraient purifiées et recyclées sur place ; des eaux usées contenant tel ou tel sous-produit pourraient être valorisées dans un autre contexte productif ; et, sur le plan quantitatif, les compteurs domestiques, le concept d'《 eau virtuelle » [Allan 2011], et des incitations économiques (tarification progressive, etc.), pourraient contribuer à une meilleure visibilité du caractère limité et territorialisé de la ressource en eau.

Néanmoins, conformément à l'idée selon laquelle « être humain 》 signifie habiter une " clairière " où les choses peuvent apparaître comme ceci ou comme cela, il serait essentiel de maintenir des "agoras », c'est-à-dire des espaces ouverts et publics (places, marchés, lieux d'assemblée politique, espaces juridiques, colloques, etc.), où toutes ces transformations dans notre rapport à l'eau et au milieu urbain peuvent s'ouvrir à des échanges, à des interprétations, et à des débats, condition préalable de toute forme de catégorisation des choses et de toute décision démocratique concernant le développement de la ville. Bref, la redécouverte de l'eau en ville ne saurait se réduire à une sorte de «spectacle » [Debord, 1967], organisé à huis clos par des gestionnaires et des technocrates - peut-être même en mimant un écosystème de type III - et dont les diverses modalités seraient établies en dehors de tout débat et de toute participation démocratique.

\section{GESTION INTEGREE}

Comme l'a bien montré le géographe canadien, Jamie Linton [2010], à la base de l'hydrologie scientifique se trouve le concept du cycle hydrologique. Ce concept est également fondamental à la Gestion Intégrée des Ressources en Eau (GIRE), car il permet de gérer les divers phénomènes hydrologiques en tant qu'éléments d'une totalité systémique. De même, le concept de " cycle hydrologique urbain " [Marsalek et al., 2008] permet de gérer de manière concertée le captage, la potabilisation, l'acheminement, l'usage, l'épuration, l'infiltration, et le stockage de l'eau en ville, d'où le concept de la " gestion intégrée des eaux urbaines ». De façon générale, celle-ci est considérée comme un élément clé du nouveau paradigme de l'hydrologie urbaine [Novotny and Brown, 2007 ; Andrieu et al., 2010 ; Chocat, 2013].

Sur le plan épistémologique, la GIRE s'inscrit dans la tradition systémique, qui remet en cause la pensée analytique traditionnelle, laquelle isole les divers éléments du monde pour les étudier et les gérer de manière séparée [von Bertalanffy, 2009]. Or, en raison de nos analyses précédentes, il nous semble que cette remise en cause de la pensée analytique n'est pas tout à fait adéquate dans le contexte de l'hydrologie urbaine. A travers la métaphore de l'organisme, la ville était déjà pensée comme un système, mais c'était un système qui fonctionnait de manière descendante à partir d'une intelligence centrale, comparable au cerveau, et représentée au niveau national par l'Etat, et au niveau de la ville par le gouvernement municipal. Les diverses entités impliquées dans la gestion de l'eau urbaine étaient donc tenues de répondre avant tout à des pouvoirs centraux, d'où, peut-être, l'impression qu'elles avaient d'être isolées les unes des autres. Ce constat est en accord avec l'observation de P. Brown [2007], selon laquelle un principe cybernétique de « command and control» (commandement et contrôle) a évolué à travers l'histoire pour émerger comme principe clé du paradigme actuel. ${ }^{7} \mathrm{Il}$ en découle que le problème fondamental de la ville moderne n'est pas qu'elle est constituée d'éléments isolés, traités de manière analytique, qui ne font pas système. Le problème est plutôt le suivant : conformément au principe cybernétique de commandement et contrôle, la dominance de canaux de communication descendants fait que les soi-disant «acteurs» locaux ne peuvent en réalité qu'exécuter les commandes issues de pouvoir centraux ou, dans le cas où ils ne sont pas d'accord avec cette commande, "rétroagir » de façon négative. La gestion de la ville se réduit donc à l'opération d'un " servomécanisme » ou, pour reprendre un jeu de mots d'Edgar Morin [1977, p.241-243] particulièrement adapté au cas de l'organisme urbain, à un " cerveau-mécanisme ». La conséquence en est qu'il manque à l'organisme urbain deux éléments organisationnels clés d'écosystèmes complexes : (i) la possibilité de véritables initiatives ou actions locales, capables de faire émerger de nouvelles propriétés à des niveaux plus globaux ${ }^{8}$; (ii) des communications latérales entre les divers acteurs locaux, et donc, en ce qui nous concerne, entre les divers acteurs du cycle hydrologique urbain, ainsi qu'avec tous leurs associés dans d'autres secteurs, tels que l'agriculture ou l'énergie, d'où la possibilité de mieux penser des concepts hautement intégrés comme le «Water-Energy-Food-Climate nexus ».

Ainsi, à la différence de l'imaginaire de l'organisme lequel, à travers la cybernétique, a su évoluer d'une simple machine cartésienne à un système puissant de commandement et contrôle -, le nouvel imaginaire de la «ville forêt " peut inspirer des actions ou des initiatives ascendantes ou latérales. Afin de mieux comprendre comment cela pourrait fonctionner dans le contexte de l'hydrologie urbaine, considérons brièvement l'analyse du «pouvoir latéral » proposée par Jeremy Rifkin dans The Third Industrial Revolution [2011]. Selon J. Rifkin, les révolutions industrielles se produisent lorsqu'il émerge un changement paradigmatique concernant à la fois la communication et l'énergie. Ainsi, alors que le paradigme dominant du $\mathrm{XX}^{\mathrm{e}}$ siècle était le réseau centralisé de génération et de transmission d'information (journaux, radio, télévision) et d'énergie (fossile, nucléaire, hydro-électrique), J. Rifkin propose

7. Cette vision cybernétique du paradigme actuel n'implique pas une forme de totalitarisme. Les pouvoirs centraux peuvent très bien être des élus tenus à répondre à des demandes locales : approvisionnement en eau potable, éloignement des eaux usées et pluviales. Néanmoins, demander quelque chose n'est pas agir ou participer à l'action, mais plutôt demander que quelqu'un d'autre agisse.

8. Les auteurs du «Genius of biome report » notent que la nature a tendance à employer « beaucoup de petits » («lots of littles ») pour résoudre des problèmes : « de nombreux éléments individuellement faibles combinent pour faire émerger un processus heuristique puissant » (2013, p.59, nous traduisons). 
comme paradigme du $\mathrm{XXI}^{\mathrm{e}}$ siècle une forme décentralisée et distribuée de génération et de transmission d'information (internet, réseaux pair-à-pair...) et d'énergie (micro-génération d'énergie renouvelable transmise à travers des réseaux bidirectionnels). Les divers acteurs de la ville (entreprises, citoyens, organisations publiques...) ne seraient plus, dès lors, de simples consommateurs d'énergie, mais aussi des producteurs d'énergie qui pourraient soit consommer leur propre énergie soit la vendre sur le réseau. En tant que producteur d'énergie, chaque bâtiment serait donc « comme un arbre ", lequel pourrait également être connecté à d'autres bâtiments par un réseau que l'on pourrait comparer à un système de rhizomes. En effet, le rhizome étant vecteur à la fois de la multiplication des modules et de la transmission et du stockage de l'énergie, il semble être un modèle ou une source d'inspiration biologique parfaitement adapté à la « Troisième Révolution Industrielle ».9

$\mathrm{Si}$, comme le veut J. Rifkin, il va falloir transformer tous les bâtiments en des " micro-centrales de génération d'énergies renouvelables » [2011, p.37] à connecter entre elles par des réseaux bidirectionnels, il faudra une gestion intégrée complexe, qui mettrait en communication des acteurs bien divers. Le contraste avec le paradigme précédent est frappant. Dans la deuxième moitié du $\mathrm{XX}^{\mathrm{e}}$ siècle, le gouvernement français a pris le choix du nucléaire - programme qu'il a réussi à faire accepter de manière descendante (ou dirigiste) par la plupart des français -, ce qui a nécessité un investissement massif dans la recherche et le développement d'une technologie extrêmement pointue et dangereuse, ainsi que la construction de grandes centrales ayant pour but de fournir de l'énergie à des usagers/consommateurs à travers des grands réseaux unidirectionnels. Dans un tel paradigme, les usagers/consommateurs ne participent guère au processus de génération et de transmission de l'énergie, lequel se fait loin de chez eux par des techniciens avec lesquels ils n'entrent pas en contact. Dans le nouveau paradigme que propose J. Rifkin, par contre, les réseaux distribués d'énergie renouvelable nécessiteraient des initiatives locales, et surtout la collaboration d'acteurs en position de générer de l'énergie renouvelable au niveau local (maison, immeuble, quartier...). Certes, l'émergence de ce nouveau paradigme pourrait être favorisée par le soutien de l'Etat (modifications réglementaires, investissements en recherche et développement, mesures économiques incitatives, etc.), voire même des grandes entreprises ; il n'empêche qu'il émergera grâce à des initiatives ascendantes et latérales impliquant des acteurs locaux beaucoup plus diversifiés en termes de compétences que ceux du paradigme précédent.

A priori, il n'y a pas de raison de limiter la Troisième Révolution Industrielle aux seuls secteurs de la communication et de l'énergie, voire même du transport (désormais électrique). A beaucoup d'égards le changement de paradigme préconisé par les hydrologues urbains correspond à celui que propose Rifkin, car de nombreux problèmes qui ont émergé dans le secteur de l'énergie s'appliquent aussi à l'eau : l'exploitation non-durable de réserves «fossiles ", la résilience faible et le gaspillage des grands réseaux, la vulnérabilité des sites centralisés de production, etc. ${ }^{10}$ Il n'est donc pas surprenant que les réponses proposées sont elles aussi souvent très similaires : utilisation de flux locaux,

9. Pour une vision philosophique du rhizome comme modèle biologique de réseaux latéraux et résilients, voir Deleuze et Guattari (1980).

10. Cela ne veut pas dire qu'il n'existe pas de différences importantes entre l'eau et l'énergie, notamment celles qui concernent la capacité physique qu'a un propriétaire foncier d'abstraire et de polluer une eau qui ne traverse que temporairement son terrain. installation de techniques décentralisées (récupération des eaux de pluie, micro-stations d'épuration, toitures végétalisées, surfaces perméabilisés...), usages secondaires de la ressource, partage de la ressource à travers des réseaux distribués, etc. De plus, comme dans le cas de l'énergie, ces réponses nécessiteront une véritable gestion intégrée impliquant de nombreux acteurs et parties prenantes : citoyens, associations de résidents, entreprises de toute taille, agences gouvernementales, chercheurs, consultants, etc.

Il nous semble, cependant, que le fait que la Troisième Révolution Industrielle de J. Rifkin ne tient pas compte de l'eau ne témoigne pas d'un simple oubli, mais plutôt d'un véritable problème. On peut certes penser le changement de paradigme de J. Rifkin selon des modèles biologiques (le bâtiment-arbre, le réseau-rhizome, etc.) ; il n'empêche que la révolution qu'il dessine n'intègre pas vraiment l'eau et le vivant eux-mêmes, comme le témoigne l'intérêt quasiment exclusif qu'il porte aux panneaux solaires, aux réseaux informatiques/énergétiques, aux voitures électriques, et aux imprimantes 3D. Certes, lorsque J. Rifkin tourne son attention vers des villes réelles (Rome, Monaco...), il imagine également des parcs et des jardins urbains, une production agricole locale et biologique, et des toitures végétalisées [Rifkin, 2011, p.83, 99]; mais ces idées restent absentes des « cinq piliers » de la Troisième Révolution Industrielle [ibid., p.36-38]. Or, la forêt n'est pas seulement un modèle biologique à imiter dans le contexte de la construction de machines et de réseaux non-vivants en milieu urbain. La « ville forêt " ne sera pas «verte » dans le seul sens métaphorique où ses bâtiments minéraux fonctionneraient « comme» des arbres. $\mathrm{Au}$ contraire, grâce à des techniques biomimétiques basées sur des symbioses avec des espèces non-humaines (ingénierie écologique, restauration de cours d'eau et de zones humides, etc.) la "ville forêt» pourrait être remplie d'une grande diversité de plantes, dont les arbres, de sorte que sa «verdure » ne soit pas seulement métaphorique.

\section{CONCLUSION}

Dans un article récent de Techniques, Sciences, Méthodes, B. Chocat pose la question suivante : " Pourquoi avons-nous autant de difficultés à mettre en pratique d'autres solutions, dites alternatives, qui valorisent les eaux de pluie urbaines ? » [Chocat, 2013, p.14] La réponse qu'il propose est que nous n'arrivons pas encore à faire de la gestion intégrée. A notre avis, cette réponse est à la fois correcte et radicalement insuffisante. De même que l'imaginaire de l'« organisme urbain » permettait aux urbanistes et aux industriels du XIX ${ }^{\mathrm{e}}$ siècle (Ward, Chadwick, Haussman, les Saint-Simoniens, etc.) de mettre en place leurs grands réseaux hydrauliques, conçus sur le modèle organiciste d'artères et de veines, seule l'émergence d'un nouvel imaginaire urbain - « la ville comme une forêt »-permettra le passage à un nouveau paradigme de l'hydrologie urbaine. En effet, comme nous l'avons montré, cette image permet de rassembler et de penser de manière cohérente trois éléments clés du « nouveau paradigme » de l'eau urbaine : la restauration ou l'imitation de flux hydrologiques naturels (biomimétisme); l'émergence de l'eau des tuyaux et son intégration comme élément central de nouveaux paysages urbains (éco-phénoménologie); et des initiatives locales et des coopérations latérales impliquant divers acteurs locaux (gestion intégrée).

Cependant, accepter que seule l'émergence d'un nouvel imaginaire peut faire émerger un nouveau paradigme nécessitera une remise en question de la logique technoscientifique 
qui continue à dominer l'hydrologie urbaine. En effet, celle-ci semble être beaucoup plus à l'aise avec des concepts conception urbaine sensible à l'eau, gestion intégrée, sécurité hydrique, nexus eau-alimentation-énergie, cycle hydro-social, etc. - qu'avec des images. Or, penser que l'hydrologie urbaine peut se passer d'images et concevoir un nouveau paradigme aves des seuls concepts n'est pas une position qui peut être justifiée par la science hydrologique elle-même ; au fond, c'est une supposition philosophique, quoique rarement formulée de manière explicite. Par ailleurs, dans la mesure où la ville du $\mathrm{XIX}^{\mathrm{e}}$ siècle, dont nous sommes aujourd'hui les héritiers, était elle-même conçue « comme » ou " par analogie » avec un organisme, et plus précisément par analogie avec un corps animal, le danger se présente que résister au nouvel imaginaire de la " ville forêt » nous condamnera à nous laisser - sans guère le savoir ou le comprendre - déterminés par ce que P. Ricoeur appelle une "métaphore morte », laquelle continuera à influencer même nos actions les plus opérationnelles par sa «fécondité occulte ». L'organisme urbain est en train de mourir. Il reste à voir si nous mourrons avec lui ou si, au contraire, nous participerons à la naissance d'un nouvel habitat urbain : la « ville forêt ».

\section{REMERCIEMENTS}

Je voudrais remercier Lyonnaise des Eaux pour leur soutien de la Chaire Industrielle : "Rationalités, Usages et Imaginaires de l'Eau » de la faculté de philosophie de l'Université Jean Moulin Lyon 3, dans laquelle ces recherches ont été effectuées.

\section{RÉFÉRENCES}

\section{Allan T. (2011) - Virtual water. I.B. London}

Andrieu H., Bruno T., Et Sylvie B. (2010) - Hydrologie urbaine, ou Comment rendre l'eau transparente dans la ville. Ecologies Urbaines. Coutard O., Lévy J.-P. (Ed.). Editions Economica. 130-139

BeAtley T. (2011) - Biophilic cities: Integrating nature into urban design and planning. Island Press

BENYUS J. (1997) - Biomimicry: Innovation inspired by nature. Harper Perennial

Bertrand-KrajewsKi J.-L. (2006) - Partie 1: Introduction générale. http://jlbkpro.free.fr/teachingmaterial/oshu3-01-introduction-generale.pdf (consulté le 14.04.2014). Cours d'hydrologie urbaine

BIOMIMICRY Group, HOK (2013) - Genius of biome report. http://issuu.com/hoknetwork/docs/geniusofbiome? $e=3095950 / 2547879$ (consulté le 23.02.2015)

Braungart M., Mcdonough W. (2009[2002]) — Cradle to cradle: Re-making the way we make things. Vintage

Brown C., Toadvine T. (2003) - Eco-phenomenology: Back to the earth itself. New York Press

BRown P. (2007) - Water resources and land use: Creating sustainable cities of the future. $R$. Camp Lecture. Kindle Edition

Сносат В. (1997) — Assainissement. L'Encyclopédie de l'assainissement et de l'hydrologie urbaine. Chocat B. (Ed.). Tec \& doc Lavoisier

Сносат B. (2013) - Un nouveau paradigme pour les eaux pluviales urbaines. Techniques, Sciences Méthodes. 6 14-15
Debord G. (1992[1967]) — La Société du spectacle. Gallimard

Deleuze G., Guattari F. (1980) — Mille Plateaux : Capitalisme et Schizophrénie 2. Les Editions de Minuit

Descola P. (2006) - Par-delà nature et culture. Gallimard

Despommier D. (2011) - The vertical farm: Feeding the world in the $21^{\text {st }}$ century. Picador

Dicks H. (2014) - A phenomenological approach to water in the city: Towards a policy of letting water appear. Environment and Planning D: Society and Space. 32(3) 417-432

Elton C. (1927) - Animal ecology. Macmillan

Harpet C. (1998) - corps, ville, industrie. Du déchet : Philosophie des immondices. L'Harmattan

Harrouel J.-M. (1977) — Les fonctions de l'alignement dans l'organisme urbain. Dix-huitième siècle. 9 135-149

HeidegGer M. (1976) — La fin de la philosophie et la tâche de la pensée. Questions III et IV. Gallimard. 281-306

Illich I. (2005) - $\mathrm{H}_{2} \mathrm{O}$ : Les eaux de l'oubli. Oeuvres Complètes. Fayard. 2 461-541

LINTON J. (2010) — What is water? The history of a modern abstraction UBC Press

MarsaleK J., Et Al. (2008) - Urban water cycle processes and interaction. UNESCO

Morin E. (1977) - La méthode: Tome 1, la nature de la nature. Editions du Seuil

Mumford L. (1961) - The city in history. Houghton Mifflin Harcourt

Musso P. (1997) - Télécommunications et philosophie des réseaux. $P U F$

Narcy J.-B. (2004) - Pour une gestion spatiale de l'eau : Comment sortir des tuyaux? - Peter Lang

Newman P., Jennings I. (2008) - Cities as sustainable ecosystems: principles and practices. Island Press

Novotny V., Brown P. (2007) - Cities of the future: The fifth paradigm of urbanization. Cities of the future: Towards integrated sustainable water and landscape management. Novotny V., Brown, P (Ed.). IWA Publishing. XIII - XXIV

Pedersen Zari M. (2015) - Mimicking ecosystems for bio-inspired intelligent urban built environments DOI 10.1080/ 17508975.2015.1007910. Intelligent Buildings International

PICON A. (2014) — La ville des réseaux : Un imaginaire politique. Manucius

Pierron J.-P. (2012) — Les puissances de l'imagination: Essai sur la fonction éthique de l'Imagination. Cerf

Ricoeur P. (1975) - La métaphore vive. Editions du Seuil

RIFKIN J. (2011) - The third industrial revolution: How lateral power is transforming energy, the economy, and the world. Palgrave Macmillan

SCHLANGER J. (1995[1971]) — Les métaphores de l'organisme. L'Harmattan

Schuiten L. (2009) - Vegetal city. Editions Mardaga

THuAL C. (2011) - Evaluer l'urbanité: rapport final. INSA de Lyon. http://www.graie.org/OMEGA2/IMG/pdf/Rapport_Final_ Constance_Thual.pdf (consulté le 14.04.2014)

Todd N.J., Todd J. (1993[1984]) — From ecocities to living machines: principles of ecological design. North Atlantic Books

Vico G. (1993[1725]) — La science nouvelle. Gallimard

VON BeRTAlanfFy L. (2009[1969]) - General system theory. George Braziller 